\title{
Nature of the unusually long solar cycles
}

\author{
Nadezhda Zolotova ${ }^{1}$ and Dmitri Ponyavin ${ }^{1}$ \\ ${ }^{1}$ Institute of Physics, St. Petersburg State University, 198504 St. Petersburg, Russia \\ email: ned@geo.phys.spbu.ru
}

\begin{abstract}
The evolution of prolonged and unusually long solar cycles such as 23rd, 20th, and especially 4 th is considered. Why the length of the 4th cycle was exceptionally large or really composed of two short cycles? Are the prolonged solar minima can be considered as precursor of low activity of the next cycles? Resolving these puzzles seems to be very important for dynamo theories trying to explain the solar long-term variations. We propose a possible model of the butterfly diagram during unusually long and prolonged cycles, based on (i) the Gnevyshev idea of sunspot distribution over the latitudes, and (ii) the phase differences of the northern and southern hemispheric activities.
\end{abstract}

Keywords. Sun: activity, sun: magnetic fields, sun: sunspots

\section{Introduction}

Analysing the latitudinal distribution of corona intensity, the appearance of prominences, and sunspots along the cycle 19, Gnevyshev found the existence of two maxima, spaced in time by 2-3 years (Gnevyshev 1963). He wrote: "The first maximum is characterized by an increase in the intensity at all latitudes between the equator and the poles with maximum at the latitude of $25^{\circ}$. During the second maximum there was an increase in the intensity only in the equatorial regions, with a maximum at latitudes of 10-15". Late on, Gnevyshev shown that sunspots, faculae, filaments, prominences, and etc. are only the manifestations of a common process, which evolves as global pulses of activity (see Antalovà \& Gnevyshev 1983). The times at which the pulses appear in both hemispheres do not coincide. Finally, the butterfly diagram is a superposition of two or more pulses, which peak at different times at different latitudes (Gnevyshev 1977). The activity distributes round centers of pulses according to the Gauss law (Gnevyshev 1977). Pulses are seen in the photosphere, chromosphere, and corona (Gnevyshev 1967, 1977). But for sunspots the double-peak structure of the cycle is less apparent, because the sunspots emerge only in the royal zones, not over the whole solar surface (Gnevyshev \& Ohl 1966). He concluded, that there is not a gradual progression of a single maximum of activity from the high latitudes to equator, as it should be according to the Spörer's law (Gnevyshev 1966, 1977). But the Maunder butterfly diagram is the result of overlapping of two or more pulses. This intersection of the decreasing and increasing branches of pulses gives the artificial illusion of gradual shifting of activity equatorwards.

\section{Gnevyshev scenario of solar cycle}

In this section we visualize the Gnevyshev idea about solar cycle composed from pulses of strongly different features. At first (Fig. 1a), we reproduce the two-pulses scenario as a mixture of bivariate Gaussian distributions, separately in hemispheres. Parameters for this two-component model (listed in Table 1) are a location on coordinate grid, covariance (fits a shape and slope of distribution), and amount of random points for distribution. 
Table 1. Parameters for the two-component model for the each hemisphere.

\begin{tabular}{|c|c|c|c|c|}
\hline & Northern 1 & hemisphere & Southern & hemisphere \\
\hline Components No. & $\| 1$ & 2 & $\| 1$ & 2 \\
\hline Latitude & \begin{tabular}{||l}
$25^{\circ}{ }^{1}$, \\
time of maximum - \\
3 years after \\
the cycle onset.
\end{tabular} & $\mid \begin{array}{l}10^{\circ}, \\
\text { time of maximum - } \\
6 \text { years after } \\
\text { the cycle onset. }\end{array}$ & \begin{tabular}{|l}
$-25^{\circ}$, \\
time of maximum - \\
4 years after \\
the cycle onset.
\end{tabular} & $\mid \begin{array}{l}-10^{\circ} \\
\text { time of maximum - } \\
7 \text { years after } \\
\text { the cycle onset. }\end{array}$ \\
\hline Covariance $^{2}$ & $\| 1 / 50$ & $\mid 2.5 / 22$ & $\| 1.5 / 50$ & $1.5 / 22$ \\
\hline Amount of points ${ }^{3}$ & $\| 3000$ & 3000 & $\| 2000$ & 3500 \\
\hline
\end{tabular}

Notes:

${ }^{1}$ The center of distribution is located at latitude $25^{\circ}$. The maximum is reached in 3 years after the cycle onset.

${ }^{2}$ Covariance is chosen as a scales ratio between $x$-y-axis. The slope was not used.

${ }^{3}$ Note, the total nominal number of sunspots, calculated for the northern hemisphere of the cycle 23 , is about 10170 counts, for the southern hemisphere - about 11450 counts.

Parameters were specified in such a way to reproduce symmetrical ascending and descending branches of a cycle, and the Gnevyshev gap (Fig. 1b). We defined the constant phase lag (equal to year) between the north and south, and different magnitude of pulses (amount of points). Figure 1b shows only temporal representation. It is analog of sunspot area (or sunspot number) time-series. Blue line corresponds the northern hemisphere, red - the southern one, and black - their sum (total). The length of this simulated double-peak cycle is about 10-11 years (Fig. 1b).

Specifying the distributions parameters and mixture proportions, the model can be modified. Levels of the hemispheric phase and amplitude asymmetries, time interval
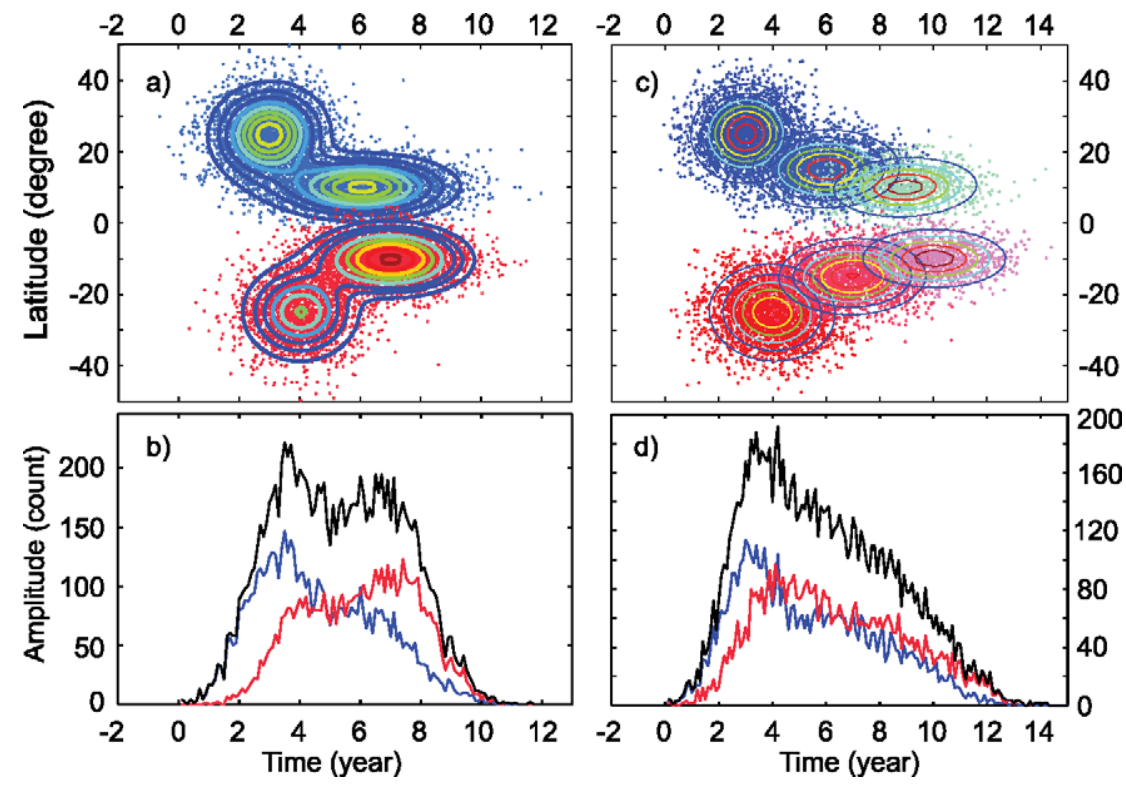

Figure 1. (a): Toy butterfly diagram composed of 4 pulses of activity. Solid lines represent levels of equal point density. (c): The same for 6 pulses. (b and d): Temporal evolutions of the same distributions summarized over latitudes. Analog of sunspots area time-series. Blue line corresponds to the northern hemisphere, red - the southern one, and black - their sum (total). 
Table 2. Parameters for the three-component model for the each hemisphere.

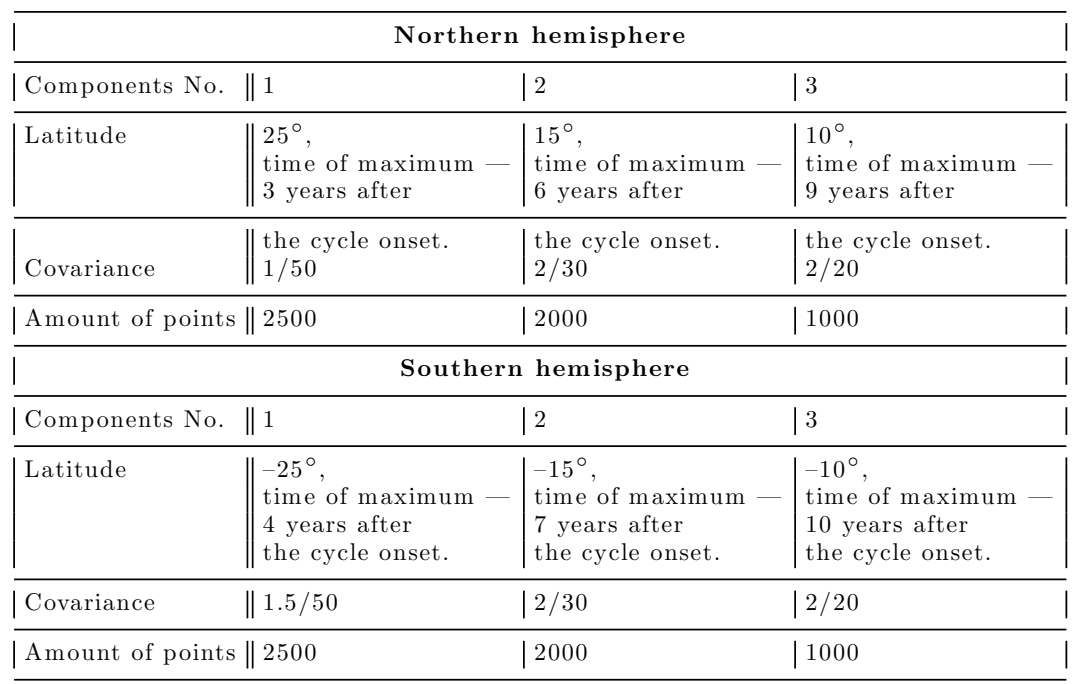

between the pulses, nonlinear stretch of distributions in time and latitudes, etc. will give a various butterfly wings geometry, different lengths and magnitudes of designed cycles.

To model prolonged cycles we added additional weak pulse in each hemisphere (Fig. 1c). Chosen parameters are listed in the Table 2. The length of this modeled doublepeak cycle is about 13-14 years (Fig. 1d). Note that the length of the 4th cycle also is unusually long (13 year, from minimum to minimum). Parameters were specified to produce one-peak maximum of final black line (Fig. 1d). It is remarkable that the declining phase became longer than the ascending one. Only just from examining the Figure 1(d), one cannot recognize that the model consist of 3 pulses in each hemisphere. Thus, the overlapping hides internal structure of the cycle. If the cycle is composed of more additional weak impulses, then its shape became even more sophisticated.

Analysing the reconstructed butterfly diagram from the Staudacher drawings (Arlt 2008, 2009a,b) for the 4th cycle (1784-1798), Usoskin et al. (2009) found that the sunspot

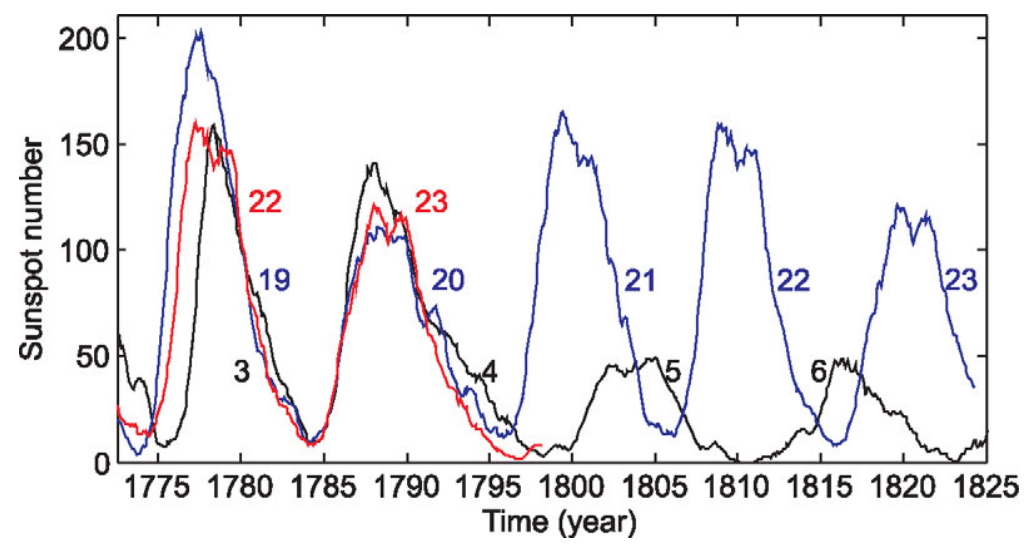

Figure 2. Smoothed Sunspot Numbers. The cycles from 3 to 6 are shown in black colour. $\mathrm{X}$-axis is time for those cycles. The cycles 19-23 - blue colour and $22-23$ - red colour. 
occurrence rate in 1793-1796 is consistent with a typical ascending phase of the solar cycle.

Hoyt \& Schatten (1998) stated that from 1795 to the present, the Group Sunspot Numbers (GSN) are well determined. From 1797 to the middle 1800 the sunspot activity was low. It was prolonged 3 -year minimum. That is why the length of the cycle 4 , determined from maximum to maximum, is 17 years. During the nowaday prolonged minimum between the cycles 23 and 24 there were already more then 800 spotless days. Hence, the 23rd cycle length from maximum to maximum should be also larger than usual (Fig. 2).

Figure 2 shows the smoothed monthly averages of Sunspot Number index. It is seen that the cycles 4, 20 and 23 are not strongly different in their shape, length and magnitude. Total activity lines (only temporal representation, convolute on latitudes) are the result of overlapping. However, a minor activity was happened in the southern hemisphere and strong north-south asymmetry was observed at the end of cycle 23 (Bankoti et al. 2010; Zolotova et al. 2009). Correspondingly, the unusually long 4th cycle can be explained by the pulse of activity in the northern hemisphere during the descending phase. We suggest that activity minimum in 1793 can be a gap between pulses. The another opposite suggestion is that this gap is illusion because of observations lack. Then, the descending phase of the 4th cycle is gradually declining, pulses are smeared by their overlapping (like in Fig. 1d). The idea of the "lost" tiny cycle also stays as a hypothesis. Thereby, lack, uncertainty, and scarcity of historical records can dramatically change our knowledge about the dynamics of solar cycle.

\section{Conclusions}

We realized the Gnevyshev idea about the composed structure of the solar cycles from the activity pulses with different features. Specifying the model parameters one- or twopeak 11-year cycle can be constructed. It is shown that by means of weak pulses the shape of the cycles can be changed. The declining phase became longer than the ascending one. Middle symmetrical and long asymmetrical cycles were presented. It was demonstrated that using only convolution of activity (like area or sunspot number data) it is difficult to recognize pulses. Even a monotonic decay may consist of them. The overlapping of pulses hides internal structure of cycle.

We suggested that the length of the 4th cycle can be explained by the pulse or impulse of activity in the northern declining phase of the cycle. Probably, it was somewhat similar to the impulse of activity in the long cycle 23, during its southern declining phase. The prolonged minimum between the cycles 23 and 24 is similar to the prolonged minimum between the cycles 4 and 5 .

\section{References}

Bankoti, N. S., Joshi, N. C., Pande, S., Pande, B., Pandey, K. New Astron., 15, 561

Antalová, A. \& Gnevyshev, M. N. 1983, Contributions of the Astronomical Observatory Skalnate Pleso, 11, 63

Gnevyshev, M. N. 1963, Soviet Astron., 7, 311

Gnevyshev, M. N. 1966, Soviet Phys. Uspekhi, 90, 291

Gnevyshev, M. N. 1967, Solar Phys., 1, 107

Gnevyshev, M. N. 1977, Solar Phys., 51, 175

Gnevyshev, M. N. \& Ohl, A. I. 1966, Soviet Astron., 9, 765

Arlt, R. 2008, Solar Phys., 247, 399

Arlt, R. 2009, Astron. Nachr., 330, 311

Arlt, R. 2009, Solar Phys.., 255, 143 
Usoskin, I. G., Mursula, K., Arlt, R., \& Kovaltsov, G. A. 2009, Astrophys. J. Lett., 700, L154 Hoyt, D. V. \& Schatten, K. H. 1998, Solar Phys., 179, 189

Zolotova, N. V., Ponyavin, D. I., Marwan, N., \& Kurths, J. 2009, Astron. Astrophys, 503, 197

\section{Discussion}

Kitiashvili: Did you have explanation, a source of such pulses?

Zolotova: Unfortunately I don't know what can - which process can cause pulses. It was suggested it is kind of burst of magnetic flux near the tachocline. 